\title{
Non-Emergency Patient Transport Services Planning through Genetic Algorithms
}

\author{
Manuel Fogue, Julio A. Sanguesa, Fernando Naranjo, Jesus Gallardo, \\ Piedad Garrido, Francisco J. Martinez \\ University of Zaragoza, Spain \\ Email: \{mfogue, jsanguesa, fnaranjo, jesusgal, piedad,f.martinez\}@unizar.es
}

\begin{abstract}
Non-emergency Patient Transport Services (PTS) are provided by ambulance companies for patients who do not require urgent and emergency transport. These patients require transport to or from a health facility like a hospital, but due to clinical requirements are unable to use private or public transport. This task is performed nowadays mainly by human operators, spending a high amount of time and resources to obtain solutions that are suboptimal in most cases. To overcome this limitation, in this paper we present NURA (NonUrgent transport Routing Algorithm), a novel algorithm aimed at ambulance route planning. In particular, NURA relies on a genetic algorithm to explore the solution space, and it includes a scheduling algorithm to generate detailed routes for ambulances. Experimental results show that NURA is able to outperform human experts in several real scenarios, reducing the time spent by patients in ambulances during non-emergency transportations, increasing ambulance usage, while saving time and money for ambulance companies.
\end{abstract}

Keywords: Genetic Algorithms; Intelligent Transportation Systems; Non-emergency Patient Transport Services.

\section{Introduction}

Route planning is a classic problem with remarkable importance in multiple environments, with a wide range of applications in the fields of Intelligent 
Transportation Systems (Liu, 2002; Fontanelli et al., 2010; Di Lecce and Amato, 2011), autonomous robotics (Latombe, 1991; Makhal et al., 2012), aerospace environments (Hui et al., 2008; Tulum et al., 2009) and military guidance and navigation systems (Zafar et al., 2006; Lei et al., 2010; Ruuben and Kreison, 2013).

An important application of route planning involves determining optimal routes for ambulances in both emergency and non-emergency transport services. Specifically, the Non-emergency Patient Transport Services (PTS) are provided by ambulance companies for patients who do not require emergency transport. These patients require transport to or from a health facility like a hospital, but due to clinical requirements are unable to use private or public transport.

Computing optimal routes for ambulances is a non-trivial problem that depends on the number and characteristics of the available ambulances, as well as their location. Several incompatibilities may arise due to the limited number of positions available in each ambulance, the equipment included, or depending on the legislation related to patient transport and minimum service conditions in each country. The main objective in this environment is, in general, to reduce the amount of time a patient spends in an ambulance which could be negative for their comfort and produce additional related health problems.

The Non-emergency Patient Transport Services Route Planning problem could be defined as the determination of the daily schedule for each available ambulance indicating the stops to be performed during the day, including the estimated time for the ambulance to arrive to each point of the route, and the patients that should be get on or off the ambulance at the stop. Most companies perform the service assignment by means of experts that are aware of the limitations of the system and the constraints that should be addressed in order to have a feasible solution, which is often a suboptimal one since human experts are not able to test enough combinations in an adequate time. The main planning unit in this problem is called service, which includes any single transportation of patients to or from a health center or a home address. For example, a return trip of a patient from his home to a hospital for a medical 
check would require two services: transferring the patient to the hospital, and another transportation to bring him home.

To solve the limitations of traditional systems, in this paper we propose the Non-Urgent transport Routing Algorithm (NURA), a route planning algorithm for non-emergency patient transport based on two main components: (i) an evolutionary algorithm (specifically, a genetic algorithm) to assign the services to be completed during a day to the set of available ambulances, and (ii) a scheduling algorithm based on solutions provided by human experts that, given the assigned services to a specific ambulance, determines the schedule for that ambulance including the times when the ambulance should pass through each point of the route, and ensures that the schedule provided is feasible.

Evolutionary Algorithms imitate the principles of natural evolution as a method to solve parameter optimization problems. They have been successfully used to solve various types of optimization problems (Greenwood et al., 1995), since they provide an optimal solution without checking all the possible solutions, reducing the execution time drastically. In this work, we compared the results obtained by our proposal with real planning obtained by human experts working in an existing ambulance company, and proved how our algorithm is able to provide better solutions, saving the time required by the experts.

This paper is organized as follows: Section 2 reviews the related work regarding non-emergency medical transport and the Vehicle Routing Problem (VRP). In Section 3 we present NURA, our proposed Non-Urgent transport Routing Algorithm which allows automatically obtaining a complete schedule for each available ambulance including all the stops to perform during the day. Section 4 introduces the structure and main parameters of the Genetic Algorithm (GA) used to explore the solution space. Section 5 presents the scheduling algorithm used to evaluate each solution. Section 6 shows the obtained results compared to those generated by human experts. Finally, Section 7 concludes this paper. 


\section{Related Work}

In this section, we are going to deal with some some approaches that are similar to the one we are introducing in this paper. This section is divided into two subsections: In the first one, we are going to mention how non-emergency medical transport has been faced by several authors when applied to different medical services around the world. In particular, we focus in the main problems that have been addressed in this field. In the second subsection we are going to review some approaches regarding the Vehicle Routing Problem (VRP) that use genetic algorithms, apart from medical transport issues.

\subsection{Approaches for non-emergency medical transport}

Non-emergency medical transport has been faced from different points of view, giving importance to different issues each time. The first thing to have in mind is that non-emergency medical transport has to be faced separately from emergency medical transport. For example, Huggins and Shugg (2008) made clear this need, and explained how non-emergency medical transport started to be treated in a separate way from the emergency one in a specific case. Also, authors remarked that the non-emergency sector would grow in size and the scope of practice would change as the population ages, and health needs change. Despite such studies, specific non-emergency medical transportation services are not often found, so novel approaches are appearing in the last years, such as the one by Safaei (2011). The specific approach mentioned in that paper, however, did not provide details about vehicle routing problems when carrying out the patients' transport.

Further studies have analyzed the quality and safety issues that have to be taken into account when dealing with non-emergency patient transport, for instance the one by Hains et al. (2011). As this paper states, quality and safety issues relating to non-emergency patient transport services have rarely been discussed compared to the transport of emergency patients. Therefore, authors identified communication, efficiency and appropriateness as the key factors that 
are advanced as impacting on the quality and safety of non-emergency transport services. Lastly, it is worth noting that vehicular networks are having a great importance recently, and of course, they are being used in patient transport situations. However, they are mostly found in emergency transport, as stated by Lee et al. (2014). In this paper, the literature is searched for suggested methods for assisting emergency vehicles, and evaluations are used simulations to evaluate them.

Thus, from this review we can conclude that non-emergency medical transport is a relevant field, which should be treated in a separate way from the emergency one, as it owns some very specific features. Non-emergency medical transport should be made as efficient as possible so that medical care is given properly to patients who make use of such service.

\subsection{Genetic algorithms applied to the Vehicle Routing Problem}

Genetic algorithms (GAs) have found usefulness in several problems in which a complex solution must be found in a wide range of options. The Vehicle Routing Problem (VRP) is one of such problems. The basic Vehicle Routing Problem consists of a number of customers, each requiring a specified weight of goods to be delivered (Baker and Ayechew, 2003). Vehicles dispatched from a single depot must deliver the goods required, and then return to the depot. Thus, medical transport can be seen as a specific application of the VRP where patients are being transported to medical centers, instead of goods to customers.

In particular, Baker and Ayechew (2003) considered the application of a genetic algorithm to the VRP and compared it to both tabu search and simulated annealing, which are the two techniques that have usually been used to solve the VRP. In their paper, authors show that genetic algorithms are an effective approach to solve the basic VRP, although they give more value to genetic algorithms as a means of diversifying the exploration of the solution space rather than being the only way of solving the problem. This is one of several approaches that apply genetic algorithm to the VRP. Another remarkable one is the approach proposed by Prins (2004), in which the author tried to develop some 
effective metaheuristics for hard combinatorial optimization problems faced in vehicle routing. Thus, he presented a hybrid genetic algorithm for the VRP able to compete with powerful tabu search algorithms in terms of average solution cost.

There are also some approaches that focused on solving some variations of the VRP, such as the VRP modified with additional time constraints. More specifically, Hwang (2002) tried to improve a genetic algorithm in order to solve such a problem. Thus, the author found that the proposed model could be potentially efficient and useful in certain conditions. More recently, Pisinger and Ropke (2007) tried to give a solution to such problems by defining a unified heuristic. In this work, authors conclude that a mixture of good and less good heuristics lead to better solutions than using good heuristics solely.

According to this, we consider that the Vehicle Routing Problem adapted to medical transport issues is a problem that can be solved by means of genetic algorithms or similar approaches. Therefore, in the following sections we are going to explain how we have addressed the problem of non-emergency medical transport. Our solution, namely NURA, consists of a route planning algorithm for non-emergency patient transport. In particular, NURA uses a genetic algorithm to assign the services to the set of available ambulances, and provides a scheduling algorithm that, given the assigned services to a specific ambulance, determines its schedule.

\section{NURA: Non-Urgent transport Routing Algorithm}

So far, genetic algorithms have been applied to different fields and applications. In particular, we previously proposed an approach focused on traffic accidents urgent sanitary resource allocation based on multi-objective genetic algorithms (Fogue et al., 2013), and a system able to reduce the emergency services arrival time by using vehicular communications and Evolution Strategies (Barrachina et al., 2014).

In this work, we propose the Non-Urgent transport Routing Algorithm (NURA), 
a route planning algorithm for non-emergency patient transport that is able to allocate non-urgent medical transport services to a set of ambulances available. In particular, NURA uses a genetic algorithm to assign the services, and it also determines the schedule of ambulances ensuring the feasibility of the solution provided.

The main goal of our system consists on obtaining a complete schedule for each available ambulance including all the stops to perform during the day, indicating the estimated time of arrival and the patients that should be picked up or left at each stop.

Determining the route for the ambulances can be divided into two subproblems, easing their solving separately:

1. Assigning a set of services to each ambulance, achieving a complete coverage of the services to perform during the day, that is, each service is assigned to exactly one of the ambulances available that day.

2. Determining the daily schedule for each ambulance taking into account the previous assignment. It is possible to find wrong or unfeasible distributions of services, mainly due to the time necessary to travel from one point to the next of the route, and the time constraints to complete the services. These issues must be considered and avoided during the design of the algorithm.

\subsection{Input Data}

Our proposed system requires data from three sources as input to compute the necessary routes:

- Set of services to complete during the day. Each service represents the transport of individual patients, either from their homes to the corresponding health center, or the opposite trip. The essential information that should be provided for each service includes: (i) initial and final address of the service, (ii) time of the day when the service should have finished, and (iii) transport features of the involved patient, i.e., whether 
the patients are able to walk on their own, or they need wheelchairs or stretchers. These issues are critical to correctly allocate patients in each ambulance.

- Available ambulances. These data include the resources available during the day to complete the assistance routes and they will limit the possible solutions of the problem. Additionally, each ambulance is characterized by the number of seats reserved for patients able to walk, seats for patients on wheelchairs, the presence or absence of stretchers in the ambulance, and the presence of additional staff occupying seats. The available schedule of the ambulance is also required to compute the routes.

- Ambulance bases. They represent the places where the ambulances start and finish their work shifts (i.e., their headquarters). The information about their location is necessary to determine the initial and final time of ambulance use, as well as the total distance traveled by each ambulance, and hence, the total cost of the assistance operation.

\subsection{Algorithm Structure}

Following the division of the problem into two sub-problems, the algorithm is divided into two main modules:

1. Service assignment to ambulances. The distribution of services to the different available ambulances is a problem with high computational cost. As an example, given a day with 80 services to complete using 5 available ambulances (supposing that we could assign each service to any of the ambulances), it makes a total of $5^{80}$ possible combinations, i.e., $8.27 \cdot 10^{55}$ possibilities. A simple "brute force" algorithm computing and checking 10,000 combinations per second would require $2.62 \cdot 10^{44}$ years to generate all the possibilities. Obviously, this is not feasible and only a subset of the combinations can be checked in an acceptable time. As the search process must be guided by an algorithm that avoids generating inade- 
quate solutions, focusing on those providing positive results, we chose an evolutionary algorithm to fulfill these objectives.

2. Generation of ambulance daily schedules. Once the services are distributed, the route for each ambulance must be computed and its feasibility checked. The subsystem in charge of this part relies on on an algorithm based on actual information from previously generated routes provided by human experts working on the ambulance company. Our algorithm sorts the stops to minimize waiting times, while ensuring the services being assisted on time. The travel time between stops must be determined using a geographic routes generation system, e.g., Google Maps API (Gibin et al., 2008; Svennerberg, 2010), to determine whether the intermediate points can be reached in the required time.

It is worth noting that both sub-systems are not independent, since they need the information provided by each other. The genetic algorithm provides the scheduling algorithm with the potential assignment of services to each ambulance, and the scheduling algorithm provides information about the feasibility and optimality of the assignment that will be later used by the genetic algorithm to compute the fitness function of a given individual.

Figure 1 shows the structure of the system, including a module in charge of the data acquisition required for the algorithm, which could be obtained from a database or files adequately structured.

\section{Service Assignment: Genetic Algorithm}

Genetic algorithms are a sub-set of evolutionary algorithms, which are based on Darwinian theories of evolution. Given a population formed by individuals, natural selection due to the limited resources and environmental pressure increase the level of adaption of the individuals to their environment, i.e., the fittest individuals are able to survive and transfer their beneficial features to their offspring. The new individuals will compete again in the environment. 


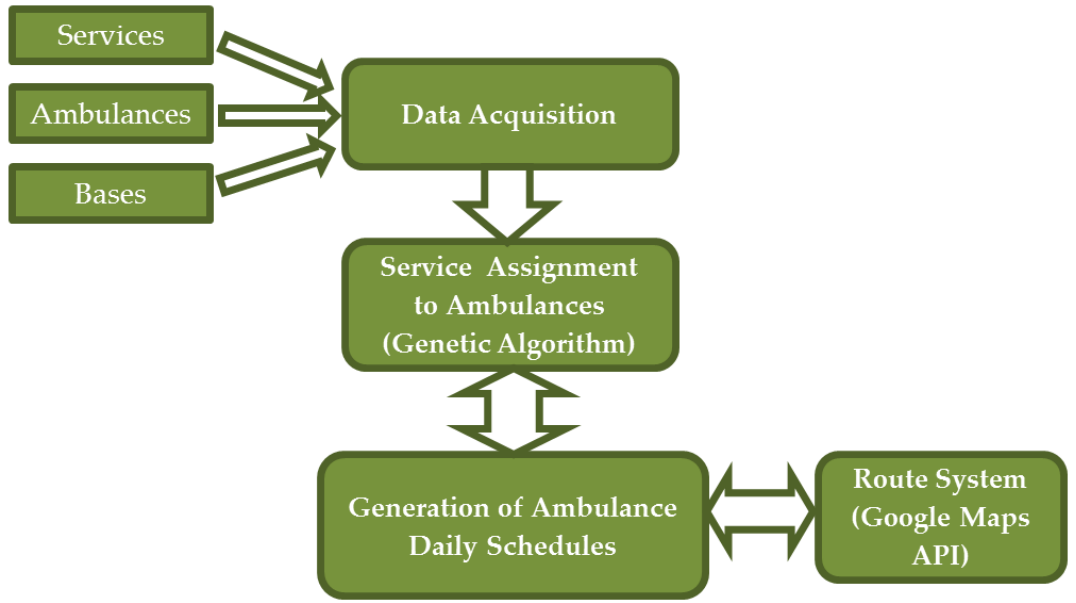

Figure 1: Structure of the NURA algorithm.

These individuals are formed by: (i) their external properties affecting their fitness or phenotype, and (ii) a set of genes codifying the corresponding phenotype or genotype (Mester and Bräysy, 2005).

Evolutionary algorithms belong to the category of search-and-test. In order to determine the level of adaptation of the individuals, also called fitness, an evaluation function estimating the quality of the solution is used. Here, fitness refers to a measure of profit, utility, or goodness to be maximized while exploring the solution space.

The evolutionary process depends on two operators: crossover and mutation. The recombination or crossover operator combines two or more genotypes (parents) to form new genotypes (offspring). Individuals with higher values of fitness are more likely to be selected as parents, allowing their offspring to inherit their genes. To increase diversity in the population, the mutation operator is in charge of introducing random changes in the chromosomes. The probability of change in a gene is usually very small to avoid excessive changes in the offspring that could move the individual away from the area that it is currently exploring, and it allows exploring new areas of the solution state space avoiding local optima. 
There are different variants of evolutionary algorithms, such as genetic algorithms, evolution strategies, evolutionary programming, and genetic programming. In the problem of assigning services to ambulances, the final solution will determine which ambulance is in charge of each service. That is, each possible solution can be represented by a vector representing the resources, and indicating which ambulance will take care of the service. Since the number of available ambulances is finite, and taking into account that GAs represent candidate solutions as strings over a defined alphabet, it makes them the most appropriate type of algorithm for our problem.

Evolutionary algorithms are able to obtain solutions with a decent quality in environments where little or none experience is available. However, in practice they are frequently applied to problems in which a considerable amount of experience and knowledge is available, and introducing heuristic methods usually improve the performance of the search process. Hybrid algorithms combining evolution and heuristics are based in the idea of "memes" (Dawkins, 1976), which are units of cultural transmission transmitted through interpersonal communication, making these hybrid approached often known as Memetic Algorithms (Moscato, 1989).

\subsection{Parameter Definition for the Genetic Algorithm}

Genetic algorithms require defining a series of components to determine their functioning and performance, and many of them are dependent on the problem to solve. In our case, we must adapt the general parameters of genetic algorithms to the ambulance routing problem.

\section{- Representation of individuals:}

Individuals representing a solution of the problem must be coded into the genetic algorithm; in particular, their genotypes, phenotypes, and the link between them. The phenotype for the ambulance scheduling problem consists on the assignment of services to each ambulance. Each individual, i.e., possible solution, is represented in the population by using a vector of characters where 


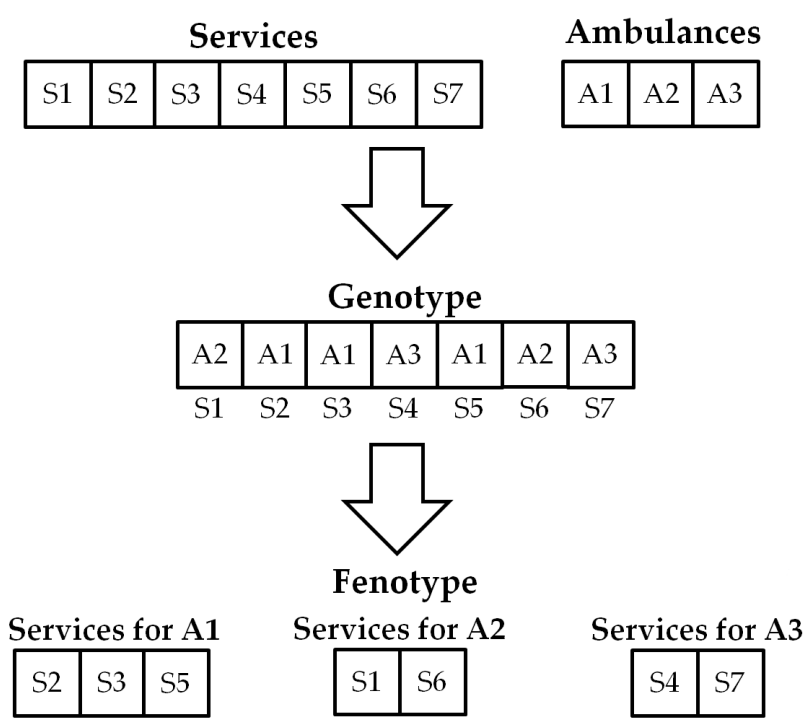

Figure 2: Example of representation of individuals in the genetic algorithm used in NURA.

each position is associated with one of the services to be performed during the day, and the value of the vector represents the ambulance in charge of the corresponding service. Due to the limited number of available ambulances, the possible symbols for each gene are finite, thus allowing the use of genetic algorithms in this scope. This representation allows the most efficient crossover and mutation, since varying the assignment of a given service only requires modifying the associated position in the genotype vector. Obtaining the set of services for an ambulance can be determined by searching all the positions in the vector in which the code of the ambulance appears. Figure 2 shows an example of assignment using a set of seven services and three available ambulances.

\section{- Evaluation function:}

The Solutions to be the parents of new generations are selected depending on their fitness values. The main objective of our system is to maximize the quality of the service provided to the patients, which can be represented by the reduction of time spent by the patients in the ambulances. We define the 
waiting time $\left(W_{T}\right)$ for a patient on a service depending on the destination of the service:

- If the destination of the service is a health facility for the treatment of the patient, the waiting time is computed as the difference between the time of the appointment and the time when patient is estimated to be picked up.

- If the destination of the service is a home address after the treatment, the waiting time is computed as the difference between the estimated time when the appointment ends and the time when patient is estimated to be left home.

We can define the fitness function as the average waiting time for all the services to be performed during the day. Equation 1 shows the computation performed to determine the fitness of an individual (ind) formed by $N$ services labeled as $s_{i}$ :

$$
\text { Fitness }(\text { ind })=\frac{100}{\frac{\sum_{i=1}^{N} W_{T}\left(s_{i}\right)}{N}+1}
$$

Therefore, the fitness value for a theoretical perfect individual without any waiting time would be 100, whereas lower values represent more unsuitable solutions to the problem.

\section{- Population:}

The population contains the candidate individuals corresponding with solutions during a generation. In genetic algorithms, populations usually contain a fixed number of individuals, because the benefits of varying the number of individuals are merely spatial (Affenzeller et al., 2007). The speed of the algorithm is affected by the size of the population, since small populations allow higher speeds but produce premature convergence of the solutions (Koljonen and Alander, 2006).

\section{- Parent selection:}




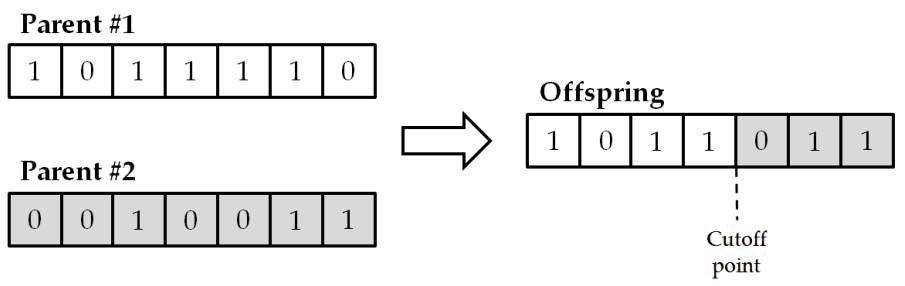

Figure 3: Example of 1-point crossover operator.

During the parent selection process, the individuals of the population are tested to determine which of them should transmit their genes to the next generation of individuals. In NURA, this phase is performed through tournament selection in which $k$ random individuals in the population are chosen for each possible father, and the best of them all becomes father of the next generation.

- Crossover operator:

The recombination or crossover operator joins the information of the parents in one or more offspring individuals. This operator is $n$-ary, even if only two parents are frequently combined. NURA makes use of the classical operator of 1-point crossover as it is the default version for this type of algorithms. Given two parent genotypes, a cutoff point is chosen and the offspring genes take values from the first parent before the point, and from the second parent after the point, as shown in Figure 3.

\section{- Mutation operator:}

The mutation operator introduces diversity in the population, and it is traditionally defined as the change probability for a single gene. NURA uses a probability of 0.05 , i.e., one change for every 20 genes on average. Mutation also takes into account the requirements of the patient to avoid assigning the corresponding service to ambulances without the necessary equipment, for example, if the transport must be performed using a stretcher and the ambulance does not include at least one.

\section{- Survival selection:}


Determining the individuals that should form the population in the new generation is not a trivial process. It is usual to use generational replacement, where the new offspring completely replaces the individuals from last generation. However, NURA uses a steady-state scheme where only a fraction of the individuals are replaced (those with the lowest fitness values) to ensure the best values obtained so far survive in future generations. In our approach, the fraction of individuals replaced in each generation is set to $50 \%$.

\subsection{Constraint Handling}

In most engineering problems, not all the possible solutions of a problem are feasible due to limited resources or other constraints. Hence, using only the fitness value of two solutions is not enough to compare them, and new techniques should be used to compensate this limitation. An interesting approach (Deb et al., 2002) makes use of the constraint-dominance concept, where a solution $i$ is fitter than (dominates) another solution $j$ if any of these conditions is true:

1. Solution $i$ is feasible, while solution $j$ is not.

2. Both solutions are unfeasible, but solution $i$ presents less violation to the feasibility condition.

3. Both solutions are feasible, but solution $i$ dominates over $j$.

Using this approach, we can compare feasible and unfeasible solutions during the search process. In NURA, a solution is considered unfeasible if one of the following conditions is fulfilled:

- One of the services assigned cannot be completed due to inability of the ambulance to arrive on time to the health center. Ambulances with too many services assigned may be unable to complete the trip on time, due to long routes or being too far away in the moment they are needed. A good example is found if an ambulance has more services assigned for a trip than its actual capacity, hence the ambulance would make two trips in order to complete them all with the subsequent loss of time. The feasibility violation of an individual is obtained as the total delay time for all the 
patients, computed as the difference between the time when the patient should arrive and the moment when he/she actually arrives.

- The duration of a patient's trip exceeds more than 1 hour the time required to reach its destination in a direct trip. That is, the additional stops added to a trip to pick up or leave a patient should not delay another patient's trip more than 1 hour. This condition follows the Spanish legislation regarding non-urgent patient transport (Ministerio de Sanidad, Servicios Sociales e Igualdad (Spain), 2012).

\subsection{Hybridization in NURA}

Hybrid or memetic algorithms make use of local search in evolutionary algorithms to reduce the level of randomness in the search process, adding knowledge about the problem to improve the efficiency of the search. In our case, local search is used to increase the quality of the solutions found when the search process has achieved almost feasible solutions. When more than $90 \%$ of the services included in the solution are assigned to suitable ambulances, the local search focuses in the rest of services, trying all the possible ambulances for them. Generating neighboring solutions to the problem at hand can be simple using gene modification, since the new solution only differs from the original one in one gene: a service is assigned to a different ambulance. The local search process will finish when the first better solution is found in the vicinity, or when there are no more neighbors left to explore.

Table 1 contains a summary of the values selected for the different parameters of the genetic algorithm proposed in NURA.

\section{Schedule Generation Algorithm}

Obtaining the fitness value for a particular solution requires knowledge of the average travel time per patient, which can only be obtained if the daily scheduling of each ambulance is known. This is done by knowing the characteristics of the particular ambulances and the services that are assigned to them for 
Table 1: Parameter Values of the Genetic Algorithm used in NURA

\begin{tabular}{|l|c|}
\hline \hline Parameter & Value \\
\hline Representation of individuals & $\begin{array}{c}\text { One gene for each service to complete } \\
\text { Everage patient waiting time [0..100] } \\
\text { Population }\end{array}$ \\
Parent selection & Fixed size \\
Crossover operator & Tournament selection size $k$ \\
Mutation operator & 1-point crossover \\
Survival selection & Probabilistic bit-flipping \\
Constraint Handling & 50\% steady-state \\
Hybridization & Constraint dominance comparison \\
\hline \hline
\end{tabular}

the day. The genetic algorithm assigns a subset of services to each ambulance, and then a method to determine the schedule of each ambulance is required to compute necessary metrics such as the patients' waiting time and the usage of the ambulances.

In NURA, the ambulance scheduling process is based on the real procedure followed by human experts in an actual ambulance company. The process followed for planning consists of a series of steps until all services are distributed in ambulance trips:

1. Step 1: Sort. The services are sorted according to the time of day. In the case of services in which the destination is a hospital, the time to consider is the time when the patient should arrive to the health facility (arrival time). If the service represents a transfer to the patient's home, the arrival time is not as important as the departure time, since more time waiting in a hospital for an available ambulance would reduce the quality of the service.

2. Step 2: Group. Once the services are sorted, they must be grouped into trips. The trips determined by the experts have their destination in the 
same city, even if the health facility is not the same. Ambulance trips should contain a number of patients not exceeding the capacity of the ambulance, but also minimizing the length of the itinerary and ensuring that the arrival time is not compromised by picking up additional passengers. Hence, all the combinations of the candidate services are considered to find the most adequate groups of services for the given ambulance.

3. Step 3: Time scheduling: After the groups of patients for a trip are obtained, the specific stops for a trip and the corresponding times are computed. The reference for trips with hospitals as destination are determined by the earliest arrival time of the patients transferred, since none of them should arrive later than their appointment. However, if the destinations are patients' homes, the departure time of the ambulance is set as the latest of the times when the appointments of the patients are estimated to finish. The times are computed taking into account: (i) the travel time of the ambulance between locations, (ii) the time needed to pick up or leave each patient in their homes, set as 5 minutes on average, (iii) the time needed to pick up or leave the patients in a hospital and their transfer to the appropriate area of the health facility, set as 10 minutes on average.

These steps are shown in Figure 4 using a small set of services. Once the time scheduling is finished, it is easy to determine if a solution is feasible or not: if the ambulance is not able to reach some destination in time, if would delay all the system and thus it would be considered unfeasible. This information is used by the genetic algorithm to evaluate the solutions and guide the search process.

\section{Experimental Results}

In this section we evaluate the performance achieved with the NURA algorithm in different scenarios obtained from a real ambulance company. First of all, we will study the influence of several parameters in the efficiency of the genetic algorithm, in order to justify the values selected and presented in previous 


\begin{tabular}{|l|l|l|}
\hline Serv. 1 & Home \#1 $\rightarrow$ Hospital \#1 & 11:00 \\
\hline Serv. 2 & Hospital $\# 1 \rightarrow$ Home \#1 & $12: 10$ \\
\hline Serv. 3 & Home \#2 $\rightarrow$ Hospital \#1 & $11: 15$ \\
\hline Serv. 4 & Hospital \#1 $\rightarrow$ Home \#2 & $11: 55$ \\
\hline Serv. 5 & Home \#3 $\rightarrow$ Hospital \#2 & $16: 00$ \\
\hline Serv. 6 & Hospital \#2 $\rightarrow$ Home \#3 & $16: 25$ \\
\hline Serv. 7 & Home \#4 $\rightarrow$ Hospital \#2 & $16: 15$ \\
\hline
\end{tabular}

(a)

\begin{tabular}{|c|c|c|c|}
\hline \multirow{2}{*}{ Trip 1$\}$} & Serv. 1 & Home $\# 1 \rightarrow$ Hospital $\# 1$ & $11: 00$ \\
\hline & Serv. 3 & Home \#2 $\rightarrow$ Hospital \#1 & $11: 15$ \\
\hline \multirow{2}{*}{ rip 2} & Serv. 4 & Hospital \#1 $\rightarrow$ Home \#2 & $11: 55$ \\
\hline & Serv. 2 & Hospital \#1 $\rightarrow$ Home \#1 & $12: 10$ \\
\hline \multirow{2}{*}{ rip 3$\}$} & Serv. 5 & Home $\# 3 \rightarrow$ Hospital $\# 2$ & $16: 00$ \\
\hline & Serv. 7 & Home \#4 $\rightarrow$ Hospital \#2 & $16: 15$ \\
\hline Trip 4 & Serv. 6 & Hospital \#2 $\rightarrow$ Home \#4 & $16: 35$ \\
\hline
\end{tabular}

(c)

\begin{tabular}{|l|l|l|}
\hline Serv. 1 & Home \#1 $\rightarrow$ Hospital \#1 & 11:00 \\
\hline Serv. 3 & Home \#2 $\rightarrow$ Hospital \#1 & $11: 15$ \\
\hline Serv. 4 & Hospital \#1 $\rightarrow$ Home \#2 & $11: 55$ \\
\hline Serv. 2 & Hospital \#1 $\rightarrow$ Home \#1 & $12: 10$ \\
\hline Serv. 5 & Home \#3 $\rightarrow$ Hospital \#2 & $16: 00$ \\
\hline Serv. 7 & Home \#4 $\rightarrow$ Hospital \#2 & $16: 15$ \\
\hline Serv. 6 & Hospital \#2 $\rightarrow$ Home \#3 & $16: 25$ \\
\hline
\end{tabular}

(b)

\begin{tabular}{|c|c|c|}
\hline \multirow{3}{*}{ Trip 1} & Pick up patient at Home \#1 & $10: 20$ \\
\hline & Pick up patient at Home \#2 & $10: 30$ \\
\hline & Arrive at Hospital \#1 & $10: 50\left(11: 00-10^{\prime}\right)$ \\
\hline \multirow{3}{*}{ Trip 2} & Pick up patients at Hospital \#1 & $12: 20\left(12: 10+10^{\prime}\right)$ \\
\hline & Leave patient at Home \#2 & $12: 40$ \\
\hline & Leave patient at Home \#1 & $12: 55$ \\
\hline \multirow{3}{*}{ Trip 3} & Pick up patient at Home \#4 & $14: 55$ \\
\hline & Pick up patient at Home \#3 & $15: 25$ \\
\hline & Arrive at Hospital \#2 & $15: 50\left(16: 00-10^{\prime}\right)$ \\
\hline \multirow{2}{*}{ Trip 4} & Pick up patient at Hospital \#2 & $16: 45\left(16: 35+10^{\prime}\right)$ \\
\hline & Leave patient at Home \#4 & $17: 25$ \\
\hline
\end{tabular}

(d)

Figure 4: Schedule generation algorithm: (a) services assigned to the ambulance, (b) services after the sort step, (c) services after the group step, and (d) final result after the time schedule step. 


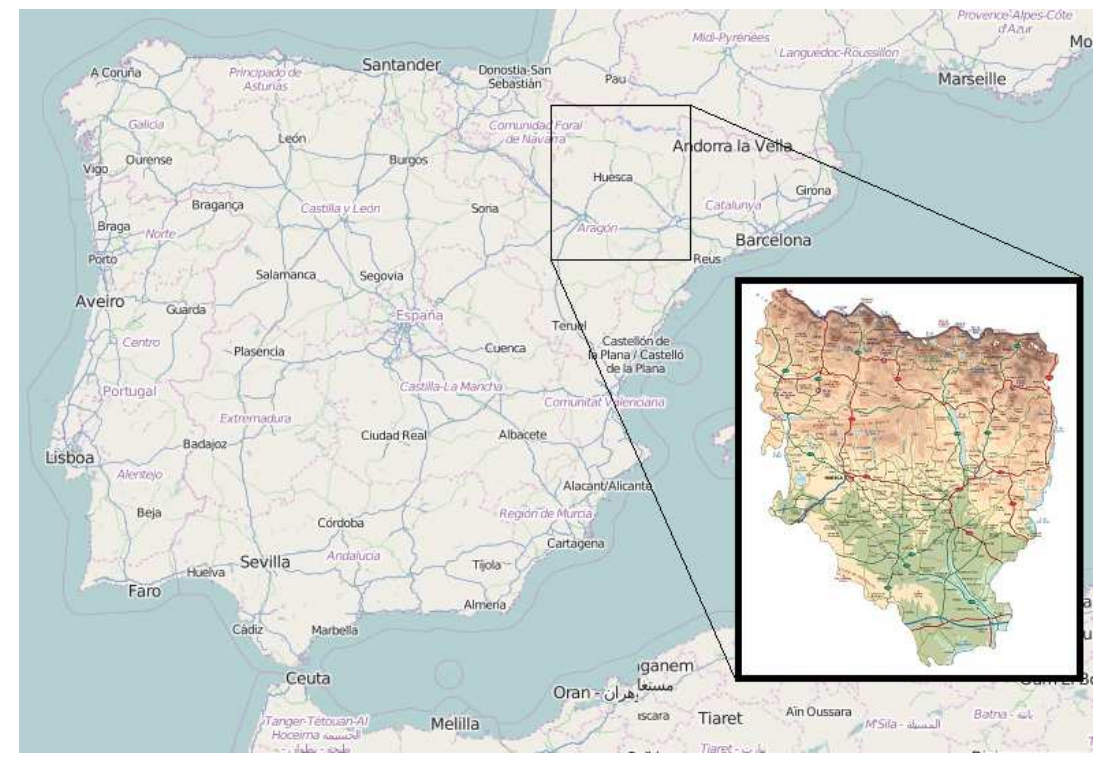

Figure 5: Region of Huesca in Spain.

sections. Secondly, we will compare the solutions generated using NURA with those computed by human experts working in the ambulance company.

Several scenarios with different amounts of ambulances and services were used to evaluate NURA, showing a similar trend in all of them. Therefore, we selected three of them representing different conditions to illustrate the performance of the algorithm. These scenarios include real services from the region of Huesca, Spain (see Figure 5), provided by the ambulance company with data about the services to be performed during the day, the ambulances available and the solution generated by experts. The characteristics of the three scenarios are shown in Table 2, including:

- Total number of services to complete.

- Number of services carrying patients from a home address to a health facility $(H A \rightarrow H F)$.

- Number of services carrying patients from a health facility to a home address $(H F \rightarrow H A)$. 
Table 2: Scenarios used to Evaluate the Performance of NURA.

\begin{tabular}{|c|c|c|}
\hline Scenario & Services & Ambulances \\
\hline Scenario 1 & $\begin{array}{c}38 \text { services } \\
19 \text { srv. } H A \rightarrow H F \\
19 \text { srv. } H F \rightarrow H A \\
14 \times \dot{\xi}\end{array}$ & $\begin{array}{c}4 \text { ambulances } \\
2 \times \text { amb. } 4-2-0 \\
2 \times \text { amb. } 3-2-0\end{array}$ \\
\hline Scenario 2 & $\begin{array}{c}52 \text { services } \\
28 \text { srv. } H A \rightarrow H F \\
24 \text { srv. } H F \rightarrow H A \\
11 \times \text { گ } 1 \times \text { ウ }\end{array}$ & $\begin{array}{l}6 \text { ambulances } \\
4 \times \text { amb. } 3-2-0 \\
2 \times \text { amb. } 3-2-1\end{array}$ \\
\hline Scenario 3 & $\begin{array}{c}94 \text { services } \\
49 \text { srv. } H A \rightarrow H F \\
45 \text { srv. } H F \rightarrow H A \\
25 \times \text { گ } 1 \times \fallingdotseq\end{array}$ & $\begin{array}{l}10 \text { ambulances } \\
2 \times \text { amb. } 4-2-0 \\
5 \times \text { amb. } 3-2-0 \\
3 \times \text { amb. } 3-2-1\end{array}$ \\
\hline
\end{tabular}

- Number of services requiring wheelchairs and stretchers.

- Total number of ambulances available.

- Configuration of each ambulance. The configurations differ among each other depending on the number of seats, number of wheelchairs allowed, and number of stretchers included in the ambulance. The ambulance company works mainly with three different configurations, as shown in Figure 6: (i) ambulances 4-2-0 (4 seats and 2 wheelchairs), (ii) ambulances 3-2-0 (3 seats and 2 wheelchairs), and (iii) ambulances 3-2-1 (3 seats, 2 wheelchairs, and 1 stretcher).

\subsection{Influence of Parameters in Algorithm Efficiency}

The efficiency of genetic algorithms noticeably relies on the selection of configuration parameters. We tested the effect of three different parameters to 


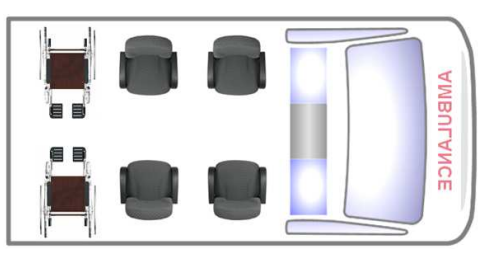

(a)

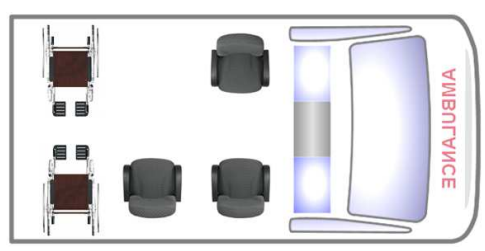

(b)

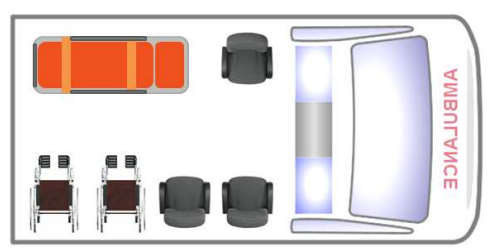

(c)

Figure 6: Different configurations of ambulances depending on the number of seats, wheelchairs, and stretchers: (a) ambulance 4-2-0, (b) ambulance 3-2-0, and (c) ambulance 4-2-1.

generate Figures 7, 8, and 9, which include the fitness values of the best individual in each generation, averaged using the data from 15 executions. These results are obtained using a fixed configuration and varying only one of the parameters to appreciate its influence in Scenario 3 (the most complex one). The basic configuration is shown in Table 3 .

The results obtained show the effect of varying the following parameters:

- Number of individuals in the population. Increasing the number of individuals allows searching a bigger portion of the search space, at the cost of increasing the computation time. We tested three different values for this parameter: 50, 100, and 200 individuals. Results in Figure 7 show that higher values increase the convergence speed of the population and the probability of obtaining feasible solutions. Since the time required to finish the computation is acceptable (90 seconds for 200 individuals, compared to 50 seconds for 100 individuals and 30 seconds for 50 individuals), the highest value is selected, i.e., 200 individuals. 
Table 3: Configuration parameters for the genetic algorithm.

\begin{tabular}{|l|l|}
\hline \hline Parameter & Value \\
\hline \hline Number of individuals & 200 individuals \\
Crossover & 1-point crossover \\
Crossover rate & $90 \%$ \\
Parent selection type & Tournament selection \\
Tournament size & $k=5$ \\
Mutation & Random bit-flipping \\
Mutation rate & 0.05 \\
Survivor selection & Steady-state model \\
Generational gap & $50 \%$ \\
\hline \hline
\end{tabular}

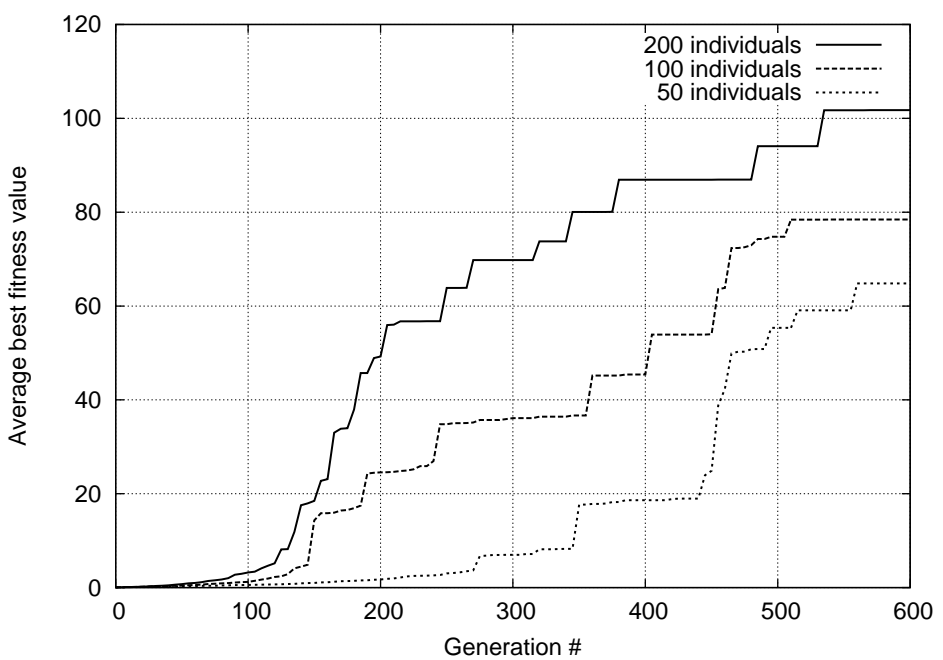

Figure 7: Evolution of the fitness value of the best individual when varying the number of individuals in the population. 


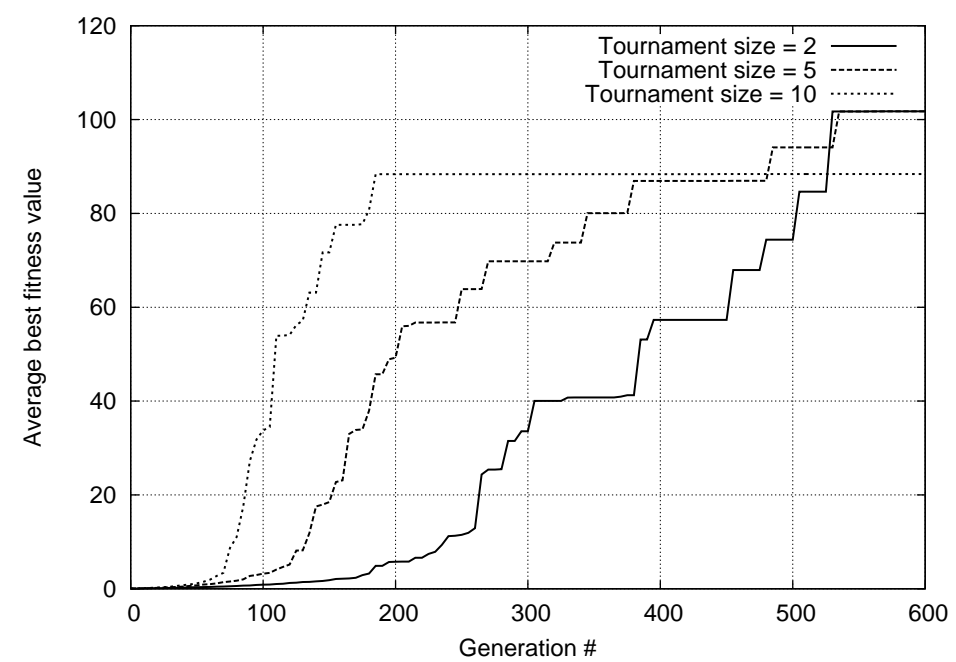

Figure 8: Evolution of the fitness value of the best individual when varying the tournament size during parent selection.

- Tournament size in parent selection. The parent selection phase is responsible of selecting the most adequate parents to generate the offspring, but it could maintain enough diversity to avoid premature convergence towards local optima. Tournament size $(k)$ determines how many individuals are used to select each parent; using low values reduces the probability of selecting good parents and slows the search process, whereas high values makes the offspring too similar with each other. Figure 8 shows how the lowest value of $k=2$ provides good solutions but the search process develops too slowly, and the value of $k=10$ accelerates the search but reduces the quality of the solutions found. The best trade-off between speed and quality of solutions is obtained when $k=5$.

- Mutation rate. Mutation is the mechanism used to maintain the diversity in the population; without it, the individuals would soon converge to the closest optima, which are often local optima. As shown in Figure 9, selecting low values of mutation rate around 0.01 makes the search process advance very fast towards the final solution, but at the cost of finding 


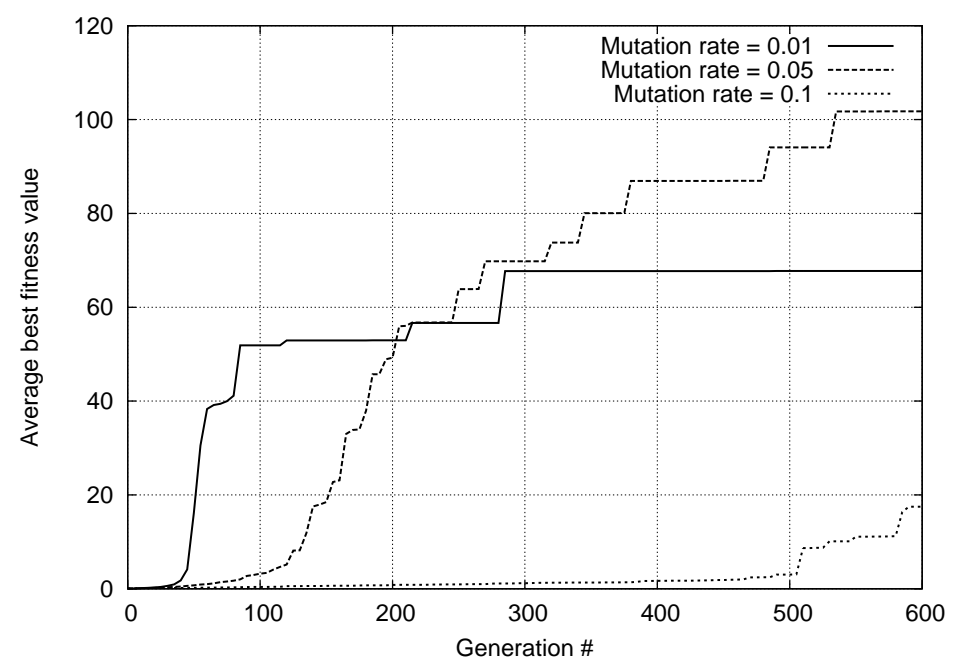

Figure 9: Evolution of the fitness value of the best individual when varying the mutation rate of the offspring.

suboptimal points. Higher values are necessary, but values higher than 0.1 makes the solutions move too far away from the area currently exploring, slowing the overall process. The best value tested for the mutation rate is 0.05 , providing enough speed to achieve good solutions with an adequate quality.

\subsection{Performance Evaluation}

We compared the results of NURA with the solutions found by the human experts of the ambulance company in the scenarios evaluated. Two metrics were tested: (i) the average waiting time of all the patients with services associated during the day, obtained as the time spent in the ambulance since the time they are picked up until they arrive to their destination; and (ii) the average usage of ambulances measured in percentage, computed as the fraction of the time the ambulances are in transit.

All the results included in this section represent an average of over 15 executions, obtaining for all of them a confidence degree $99 \%$. 


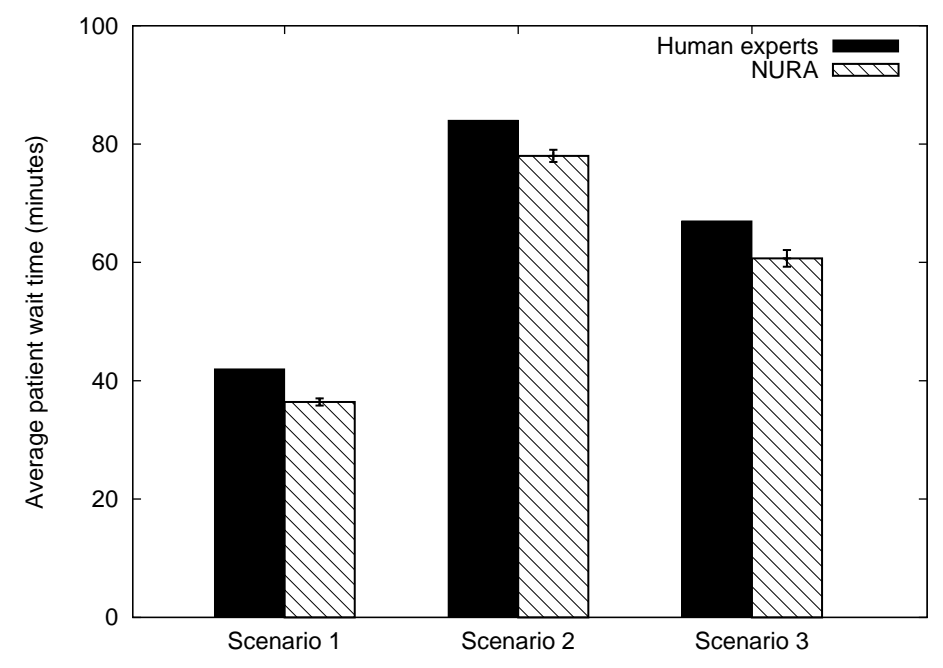

Figure 10: Average waiting time for patients in the solution found by human experts and NURA.

Figure 10 shows the results regarding average waiting time when comparing both the human experts and NURA solutions. It is noticeable how Scenario 2 is the one that presents higher average waiting time even if it is not the one with the highest amount of services and ambulances and the number of services per ambulance is the lowest (9.5 services/ambulance in Scenario 1, 8.7 in Scenario 2, and 9.4 in Scenario 3), mainly due to the complicated location of the patients which requires longer trips. NURA is able to outperform human experts in the three selected scenarios, showing remarkable performance in scenarios with low, medium and high number of services and ambulances involved.

Regarding ambulance usage, as shown in Figure 11, NURA makes a better use of the available ambulances, reducing the inactive periods of the ambulances in all the scenarios tested. This could help determining the optimal amount of ambulances needed for a given set of services to be completed during the day, thereby reducing the total cost of the operation.

Table 4 summarizes the information contained in the previous figures. As it can be observed, the reduction in patient waiting time is about $10 \%$ in average, 


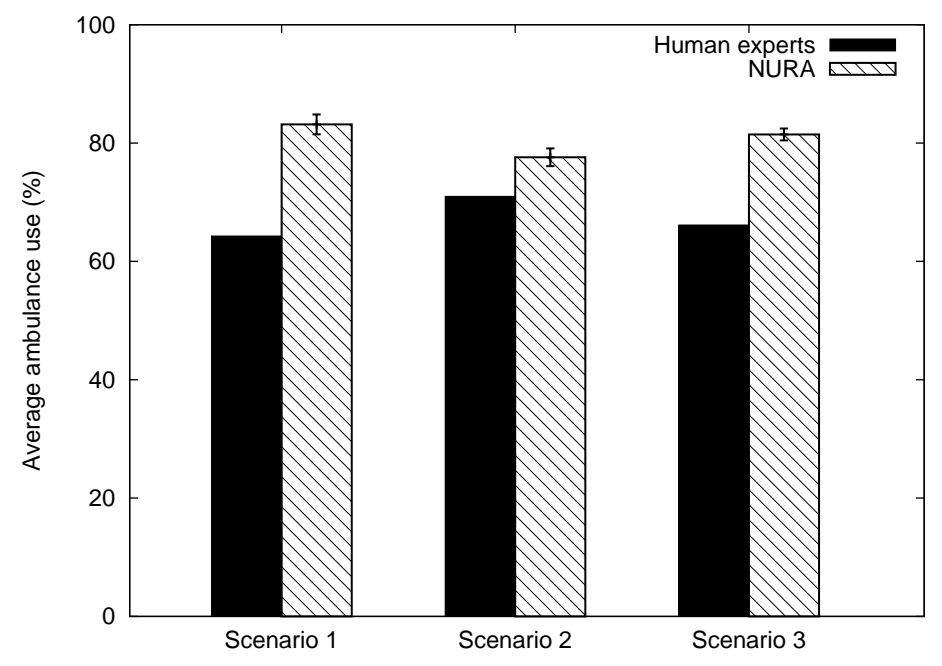

Figure 11: Average ambulance usage in the solution found by human experts and NURA.

reaching up to $13 \%$ in Scenario 1. The improvement is even higher in terms of ambulance usage, with almost $30 \%$ increased usage in Scenario 2 allowing the reduction of waiting time detected. This would reduce the incommodity of the patients during transportation, improving the perception of the users towards the service provided.

\section{Conclusions}

Non-emergency patient transport is a rapidly growing area, especially due to demographic changes in today's society. However, specific non-emergency medical transportation services are not often found, as they are mainly focused on emergency medical transport.

In this paper we presented NURA, a novel algorithm to generate NonEmergency Patient Transport routes with the aim of reducing the time spent by the patients in the ambulances. Existing ambulance companies make use of human experts to generate these routes; however, this task is time-consuming and the solutions found are in most of cases suboptimal due to the reduced 
Table 4: Performance comparison.

\begin{tabular}{|c|c|c|c|c|}
\hline \hline \multirow{2}{*}{ Scenario } & \multicolumn{2}{|c|}{ Patient waiting time } & \multicolumn{2}{c|}{ Ambulance usage } \\
\cline { 2 - 5 } & Human experts & NURA & Human experts & NURA \\
\hline \hline \multirow{2}{*}{$\mathbf{1}$} & $42 \mathrm{~min}$. & $\begin{array}{c}36.40 \mathrm{~min} . \\
(-13.33 \%)\end{array}$ & $70.98 \%$ & $\begin{array}{c}7.59 \% \\
(+9.31 \%)\end{array}$ \\
\hline \multirow{2}{*}{$\mathbf{2}$} & $84 \mathrm{~min}$. & $78.00 \mathrm{~min}$. & $64.29 \%$ & $83.16 \%$ \\
& & $(-7.14 \%)$ & & $81.44 \%$ \\
$\mathbf{3}$ & $67 \mathrm{~min}$. & $60.67 \mathrm{~min}$. & $66.14 \%$ & $(+23.13 \%)$ \\
\hline \hline
\end{tabular}

number of possibilities that can be explored in a reasonable time. NURA copes with these deficiencies using a genetic algorithm to guide the search process in the solution space, and a scheduling algorithm able to generate the specific planning for an ambulance given a set of assigned services.

Evolutionary algorithms are able to obtain suitable solutions in a reasonable time in environments where little or none experience is available. In the field of non-emergency patient transport, the number of possible solutions increases exponentially with the size of the problem; hence, a mechanism able to efficiently explore huge parts of the search space while avoiding local optima is needed. Evolutionary algorithms, and specifically genetic algorithms, fulfill these requirements. NURA additionally includes hybridization using heuristics to further improve the performance of the search process.

In addition, unlike NURA, most approaches focusing on emergency medical transport have not been tested against human experts doing this task in ambulance companies. The results obtained when comparing the solutions found by human experts and NURA under the same conditions show that our proposal is able to reduce the wait time of patients during their transport up to $13.33 \%$, while increasing the ambulance usage up to $29.35 \%$ in the selected scenarios. 
Hence, the approach followed combining genetic algorithms with an schedule generation algorithm is able to provide more efficient routes in less time than human experts, with the consequent increase in productivity and optimization of resource usage.

We are planning to further enhance NURA using a multi-objective genetic algorithm taking into account more possible outcomes of the solution, including total cost of the operation and distance traveled by the ambulances. In addition, the scheduling algorithm could be improved increasing the flexibility of the ambulance trips, allowing multiple stops at health facilities in each trip.

\section{Acknowledgments}

This work was partially supported by the Ministerio de Economía y Competitividad, Programa Estatal de Investigación, Desarrollo e Innovación Orientada a los Retos de la Sociedad, Proyectos I+D+I 2014, Spain, under Grant TEC2014-52690-R, and by the Government of Aragón and the European Social Fund (T91 Research Group).

Affenzeller, M., Wagner, S., Winkler, S., 2007. Self-adaptive Population Size Adjustment for Genetic Algorithms. In: Moreno Daz, R., Pichler, F., Quesada Arencibia, A. (Eds.), Computer Aided Systems Theory EUROCAST 2007. Vol. 4739 of Lecture Notes in Computer Science. Springer Berlin / Heidelberg, pp. 820-828.

Baker, B. M., Ayechew, M., 2003. A genetic algorithm for the vehicle routing problem. Computers and Operations Research 30 (5), 787-800.

Barrachina, J., Garrido, P., Fogue, M., Martinez, F. J., Cano, J.-C., Calafate, C. T., Manzoni, P., 2014. Reducing emergency services arrival time by using vehicular communications and evolution strategies. Expert Systems with Applications 41 (4, Part 1), 1206-1217.

Dawkins, R., 1976. The Selfish Gene. Oxford University Press, UK. 
Deb, K., Pratap, A., Agarwal, S., Meyarivan, T., 2002. A fast and elitist multiobjective genetic algorithm: NSGA-II. IEEE Transactions on Evolutionary Computation 6 (2), 182-197.

Di Lecce, V., Amato, A., September 2011. Route planning and user interface for an advanced intelligent transport system. IET Intelligent Transport Systems 5 (3), 149-158.

Fogue, M., Garrido, P., Martinez, F. J., Cano, J.-C., Calafate, C. T., Manzoni, P., 2013. A novel approach for traffic accidents sanitary resource allocation based on multi-objective genetic algorithms. Expert Systems with Applications 40 (1), 323-336.

Fontanelli, S., Bini, E., Santi, P., Dec 2010. Dynamic route planning in vehicular networks based on future travel estimation. In: 2010 IEEE Vehicular Networking Conference (VNC). pp. 126-133.

Gibin, M., Singleton, A., Milton, R., Mateos, P., Longley, P., 2008. An exploratory cartographic visualisation of London through the Google Maps API. Applied Spatial Analysis and Policy 1 (2), 85-97.

Greenwood, G., Lang, C., Hurley, S., Apr. 1995. Scheduling tasks in real-time systems using evolutionary strategies. In: Proceedings of the Third Workshop on Parallel and Distributed Real-Time Systems. pp. 195-196.

Hains, I. M., Marks, A., Georgiou, A., Westbrook, J. I., 2011. Non-emergency patient transport: what are the quality and safety issues? A systematic review. International Journal for Quality in Health Care 23 (1), 68-75.

Huggins, C., Shugg, D., 2008. Non-emergency patient transport in victoria: An overview. Journal of Emergency Primary Health Care 6 (4).

Hui, Y., Zhong, Y., Weihua, L., Dec 2008. Research on planning multiple routes for air vehicles based on niche particle swarm optimization technology. In: 2008 International Conference on Computer Science and Software Engineering. Vol. 1. pp. 1211-1214. 
Hwang, H.-S., 2002. An improved model for vehicle routing problem with time constraint based on genetic algorithm. Computers and Industrial Engineering $42(2-4), 361-369$.

Koljonen, J., Alander, J. T., 2006. Effects of population size and relative elitism on optimization speed and reliability of genetic algorithms. In: Proceedings of the Ninth Scandinavian Conference on Artificial Intelligence (SCAI 2006). pp. 54-60.

Latombe, J.-C., 1991. Robot Motion Planning. Kluwer Academic Publishers, Norwell, MA, USA.

Lee, C.-L., Huang, C.-Y., Hsiao, T.-C., Wu, C.-Y., Chen, Y.-C., Wang, I.-C., 2014. Impact of vehicular networks on emergency medical services in urban areas. International Journal of Environmental Research and Public Health 11 (11), 11348-11370.

Lei, B., Qing, X., Zheng, C., Yu, K., Oct 2010. The direction join algorithm based on GIS and its application in tank mobile route planning. In: 2010 International Conference on Computer Application and System Modeling (ICCASM). Vol. 10. pp. V10-303-V10-306.

Liu, C.-L., 2002. Best-path planning for public transportation systems. In: The IEEE 5th International Conference on Intelligent Transportation Systems. pp. 834-839.

Makhal, A., Raj, M., Singh, K., Chakraborty, P., Nandi, G., Nov 2012. Path planning through maze routing for a mobile robot with nonholonomic constraints. In: 2012 9th International Conference on Ubiquitous Robots and Ambient Intelligence (URAI). pp. 325-331.

Mester, D., Bräysy, O., 2005. Active guided evolution strategies for large-scale vehicle routing problems with time windows. Computers \& Operations Research 32 (6), 1593-1614. 
Ministerio de Sanidad, Servicios Sociales e Igualdad (Spain), 2012. Proyecto de Orden por la que se define la cartera comn suplementaria de transporte sanitario no urgente del Sistema Nacional de Salud. Available at http:// www.msssi.gob.es/normativa/docs/Otransportenourgente.pdf.

Moscato, P., 1989. On Evolution, Search, Optimization, Genetic Algorithms and Martial Arts - Towards Memetic Algorithms. Tech. rep., Caltech Concurrent Computation Program 158-79, California Institute of Technology. Pasadena, CA, USA.

Pisinger, D., Ropke, S., 2007. A general heuristic for vehicle routing problems. Computers and Operations Research 34 (8), 2403-2435.

Prins, C., 2004. A simple and effective evolutionary algorithm for the vehicle routing problem. Computers and Operations Research 31 (12), 1985-2002.

Ruuben, T., Kreison, O., Nov 2013. Route planning in asymmetric military environments. In: 2013 Second International Conference on Future Generation Communication Technology (FGCT). pp. 73-80.

Safaei, J., 2011. A ride to care - a non-emergency medical transportation service in rural british columbia. Rural and Remote Health 11 (1).

Svennerberg, G., 2010. Beginning Google Maps API 3. Apress.

Tulum, K., Durak, U., Yder, S., March 2009. Situation aware uav mission route planning. In: 2009 IEEE Aerospace conference. pp. 1-12.

Zafar, K., Qazi, S., Baig, A., Sept 2006. Mine detection and route planning in military warfare using multi agent system. In: COMPSAC'06 30th Annual International Computer Software and Applications Conference. Vol. 2. pp. 327-332. 